\title{
Creating Personalized Digital Human Models Of Perception For Visual Analytics
}

\author{
Mike Bennett ${ }^{1}$ and Aaron Quigley ${ }^{2}$ \\ 1 SCIEN, Department Of Psychology, Stanford University \\ mikemb@stanford.edu \\ 2 SACHI, School Of Computer Science \\ North Haugh, University of St Andrews, Scotland \\ aquigley@cs.st-andrews.ac.uk
}

\begin{abstract}
Our bodies shape our experience of the world, and our bodies influence what we design. How important are the physical differences between people? Can we model the physiological differences and use the models to adapt and personalize designs, user interfaces and artifacts? Within many disciplines Digital Human Models and Standard Observer Models are widely used and have proven to be very useful for modeling users and simulating humans. In this paper, we create personalized digital human models of perception (Individual Observer Models), particularly focused on how humans see. Individual Observer Models capture how our bodies shape our perceptions. Individual Observer Models are useful for adapting and personalizing user interfaces and artifacts to suit individual users' bodies and perceptions. We introduce and demonstrate an Individual Observer Model of human eyesight, which we use to simulate 3600 biologically valid human eyes. An evaluation of the simulated eyes finds that they see eye charts the same as humans. Also demonstrated is the Individual Observer Model successfully making predictions about how easy or hard it is to see visual information and visual designs. The ability to predict and adapt visual information to maximize how effective it is is an important problem in visual design and analytics.
\end{abstract}

Keywords: virtual humans, physiology modeling, computational user model, individual differences, human vision, digital human model

\section{Introduction}

Our bodies shape our experience of the world, and our bodies influence what we design. For example clothes are not designed for people with three arms because designers implicitly model standard human physiology. Yet, human bodies differ, some people are born with small bodies, others with bodies that see colors differently (colorblindness). How important are these physical differences between people? Can we model the physiological differences and use the models to adapt and personalize designs, user interfaces and artifacts?

Many domains, such as medicine, health, sports science, and car safety are creating digital human models [7]. These digital human models are very useful for identifying and evaluating the strengths and weakness in prototype artifacts and novel tools. Initially, the majority of the digital human models were primarily concerned with modeling humans' physical bodies and biomechanics [3]. 


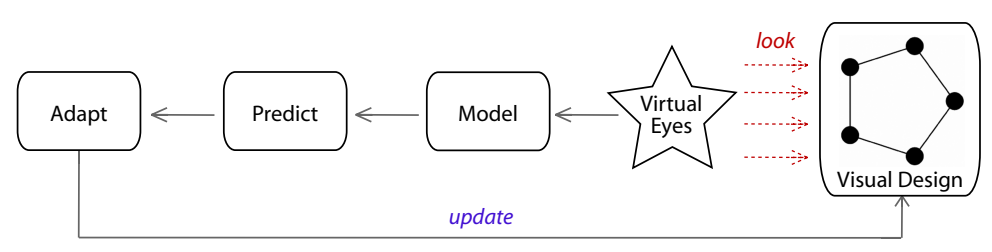

Fig. 1. To adapt a visualization or visual design we use Individual Observer Models of eyesight. The models integrate with predictors, which feed into adaption techniques for improving the layout and presentation of visualizations and visual designs.

Recently, there has been a move to richer and multifaceted digital human models, which are capable of modeling many aspects of being human, including modeling aspects of cognition, simulating affect (emotion) [12], modeling group and social dynamics, and simulating aesthetics and taste [6]. Numerous challenges and research opportunities exist for creating and integrating biomechanical models with cognitive and perceptual models $[10,16,7]$.

In this work, we create personalized digital human models of perception, particularly focused on how humans see (Figure 1). With digital human models of eyesight a visual design can be evaluated to establish what parts of a design are easy or difficult to see. For example when viewing an information visualization on a wall sized display from far away, how small can the visual features of the information visualization be before they are impossible to clearly and easily see?

Individual differences in human bodies often cause differences in how humans perceive and experience the world, e.g. colorblindness. Introduced in this paper are Individual Observer Models, which are user models of individual bodies and perceptions. Individual Observer Models capture how our bodies shape our perceptions. Individual Observer Models are useful for adapting and personalizing user interfaces to suit individual users' bodies and perceptions.

We introduce and demonstrate an Individual Observer Model of human eyesight, which we use to simulate 3600 different biologically valid human eyes. An evaluation of the simulated eyes finds that they see eye charts the same as humans. Also demonstrated is the Individual Observer Model successfully making predictions about how easy or hard it is to see visual information. The ability to predict and adapt visual information to maximize how effective it is is an important problem in visual design and analytics.

\section{Modeling And Creating Individual Virtual Eyes}

To build the Individual Observer Model of human eyesight we create a simplified optical model of how the human eye works. The model has parameters for controlling the amount of individual differences in eyesight. The eyesight model is built on research from vision science [14], optometry [13] and ophthalmology [9]. Fortunately, modeling individual differences in eyesight is extensively studied in optometry and ophthalmology research [4, 21, 8, 13].

Building models of human eyesight is challenging, both technically and because many questions remain unsolved about how human vision works. In order to build a useful human vision model, we limit how much of human vision we 


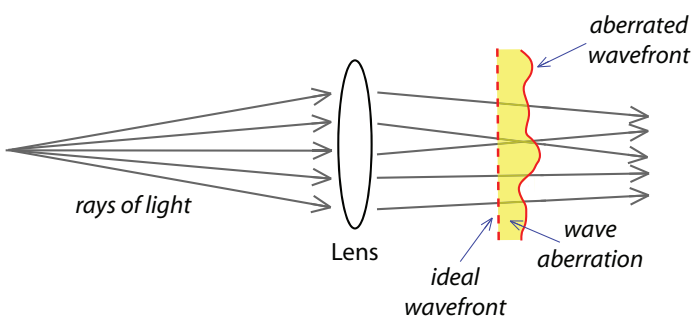

Fig. 2. Example of ideal and aberrated wavefronts generated by rays of light travelling through an optical system (eye).

model. Specifically, we focus on modeling how light travels through the human eye. Individual differences between peoples' eyes are accounted for by modeling individual differences in the physical structure of human eyes.

Depending on the physical structure of eyes, some peoples' eyes are very good at focusing light on the back of the eye, while in other cases the eyes are bad at focusing light. This difference in how well the eye does or does not focus light is due to the amount of aberrations in the eyes. People with eyes that have high amounts of aberrations usually have worse eyesight than those with low amounts of eye aberrations. Nobody has aberration free eyesight, but there are normal amounts and types of aberrations.

Differences in the amount of eye aberrations has a large impact on how easily people can or cannot see visual information. In particular, modeling eye aberrations is good for predicting the amount of visual detail people can see. The ability to see visual detail is called visual acuity. Good visual acuity commonly implies low amounts of eye aberrations, or that an eye has been corrected to reduce the impact of the aberrations. Correction is done either with eye glasses, or with various kinds of eye surgery. Visual acuity is known to significantly differ between people. In some cases this difference is genetic in origin, in other cases it is due to age related changes, and other times it is due to medical issues [21].

The ability to model individual human eyes gives us the ability to measure how individual eyes transform visual information. We can take a visual design, pass it through a virtual eye, then capture the visual design as it is seen at the back of the virtual eye.

\subsection{Modeling The Flaws And Aberrations In A Human Eye}

In this and the following subsections we briefly describe our model of the human eye and how it works. Background on the particular approach we adopt for modeling eye aberrations can be found in Krueger et al.'s work on human vision [9]. Far more vision science detail and background on our approach to modeling individual eyes and human vision can be found in [1].

Our eye model accounts for how rays of light travel through the human eye. Looking at Figure 2 you can see that multiple rays of light are entering a lens (eye). After the rays of light pass through the lens they are not aligned with each other, and in some cases mistakenly cross. In a perfect eye the rays of light are focused on a single spot (fovea), while in an aberrated eye the light 
rays are imperfectly focused. Depending on the location at which a ray of light passes through the lens, it will get aberrated in different ways and by different amounts. In order to model how different parts of the lens affect light rays, we use wavefronts. Wavefronts describe how numerous light rays simultaneously behave over many points of a lens [9]. A wavefront is perpendicular to the light ray paths.

For example, in Figure 2 we have an ideal wavefront and an aberrated wavefront. The ideal wavefront is the dotted line, and it represents all the light rays emerging from the lens in parallel. Unfortunately, all the light rays are not parallel so the wavefront is distorted and aberrated. Wavefronts are widely used by ophthalmologists when planning eye surgery to correct human vision, such as LASIK eye surgery [9].

\subsection{Simulating Individual Differences In Eyesight}

Wavefronts enable us to model individual eyes, because we can create and simulate wavefronts $\left(W_{\text {eye }}\right)$, then use the wavefronts to transform a visual design into what is seen at the back of the human eye. Provided in Equation 1 is the wavefront aberration function for modeling wavefronts. For details on using Zernike Polynomials to model human eyes see $[1,18,20,19,9]$.

The important thing to realize from the wavefront function equation is that the Zernike coefficients $\left(C_{n}^{m}\right)$ weigh the Zernike modes $\left(Z_{n}^{m}\right)$. Each Zernike mode (roughly) corresponds to a particular type of aberration commonly found in the human eye, such as astigmatism, defocus or coma. Each Zernike coefficient describes how much of each particular kind of aberration occurs. When you sum up all the aberrations you end up with a virtual wavefont $\left(W_{\text {eye }}\right)$ that describes how light is altered as it passes through the human eye.

To simulate the wavefront of an individual's eye, we sum the first fourteen aberrations (Zernike modes) and for each aberration set the amount of aberration (Zernike coefficient) by randomly picking a value from within the normal range for that type of aberration.

Elsewhere [20], it has been established what the normal values and ranges of each Zernike coefficient is. This was achieved by measuring and analysing over 2560 wavefronts of healthy human eyes [18]. We use the first fourteen aberrations

Equation 1. Wavefront aberration function as weighted sum of Zernike Polynomials [20].

$$
W_{\text {eye }}(p, \theta)=\sum_{n, m} C_{n}^{m} Z_{n}^{m}(p, \theta)
$$

where

$C_{n}^{m}$ is Zernike coefficient in microns $\mu m$

$Z_{n}^{m}$ is double indexed Zernike mode (see [19])

and

$p$ is normalized pupil radius

$\theta$ is azimuthal component from 0 to $2 \pi$ radians 
as it has also been previously established that they matter the most. Provided in [1] on page 86 Table 3.2 are the ranges of Zernike coefficients we use.

\subsection{Simulating What Is Seen At The Back Of An Eye}

Once we have a virtual eye wavefront $\left(W_{\text {eye }}\right)$, we use the virtual eye $\left(W_{\text {eye }}\right)$ to transform the original design into the design as seen by the back of the human eye. In order to transform the original design, we convert the $W_{\text {eye }}$ to an image convolution kernel (widely used in image filtering), and then apply the image kernel to the original design. The resulting image is the design as it is seen at the back of the eye.

Shown in Figure 3 are examples of how a photograph of a pair of shoes on grass is seen by three different eyes. The amount of individual differences between the eyes is small. A limitation of our current eye simulation is that it is restricted to grayscale images. This restriction exists because in vision science it is not yet known what the normal aberration amounts for color eyesight are.

\subsection{Predicting What Users Can Or Cannot See}

To predict what a user can or cannot see, we use the virtual eyes in a predictor (Figure 1). The predictor quantifies how differently individual eyes see the same visual information. Quantifying the impact of individual differences in eyesight enables us to improve the layout and presentation of visual information, by adapting it to suit individual eyes and groups of eyes.

The predictor works by looking at the original visual design through a virtual eye, then it compares the original design against the design as seen at the back of the eye. The difference between the original design and the perceived design gives a measure of how individual differences in peoples' eyesight impacts upon the perception of visual information.

In this work Perceptual Stability For Visual Acuity $\left(P E R S_{v a}\right)[1]$ is used to measure the differences between the original and perceived design. $P E R S_{v a}$ is a space-scale approach to image analysis, which uses an information theoretic measure of image content. Further details on the vision science motivation and equations for $P E R S_{v a}$ can be found elsewhere [1].

When used, $P E R S_{v a}$ gives a single value score, which indicates how differently the original and perceived design look. A high $P E R S_{v a}$ score indicates that the visual design changes a lot and is harder to see due to passing through an aberrated eye, while a low score indicates that the visual design is easier to see and does not change much due to the aberrations.
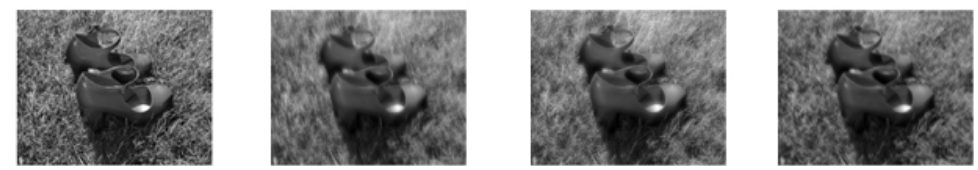

Fig. 3. Example of how three different simulated eyes see a photograph of shoes on grass. First photograph is the original version. 


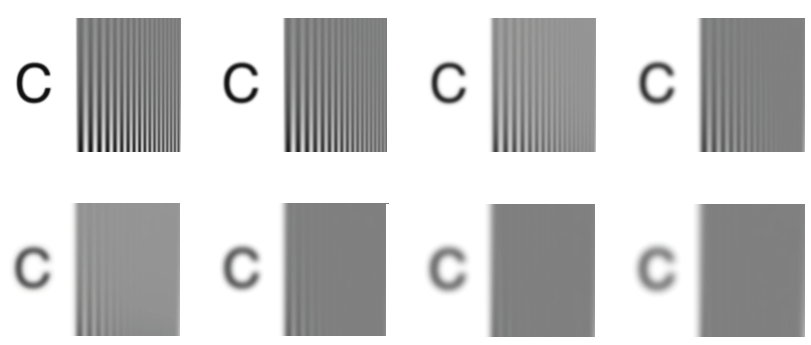

Fig. 4. Examples of how increasing amounts of aberration effects two different visual patterns. On the left is the letter $\mathrm{C}$, while on the right is a pattern of alternating bars. Amount of aberration increasing from left to right, top to bottom.

For example, if the same virtual eye looks at the two visual patterns as shown in Figure 4. For the virtual eye, the $\mathrm{C}$ pattern has a lower $P E R S_{v a}$ score than the alternating bars, which indicates that the aberrations in the eye effect the perception of the alternating bars more than the perception of the $\mathrm{C}$.

\section{Evaluation}

In order to evaluate the Individual Observer Model of human eyesight, we test whether the virtual eyes see the same as human eyesight. To establish whether the virtual eyes see the same as human observers, we generate 3600 different biologically valid eyes. Each of the 3600 virtual eyes looks at various visual patterns, such as eye charts. If the virtual eyes are valid, then they should agree with human judgements about what parts of the eye charts are easy or hard to see.

For example, shown in Figure 5 is an eye chart that is commonly used to measure how well people can or cannot see. It is known that a person looking at the eye chart will find letters at the top of the chart easier to see than at the bottom of the chart. Figure 8 shows how 300 virtual eyes judge what parts of the eye chart are easier or harder to see (the red text is the normalized PERS scores). The top of the eye chart is white, indicating it is seen easiest, while the bottom of the eye chart is black, indicating it is the hardest part of the eye chart to see.

\subsection{Eye Charts Are Gold Standard Measure Of Human Eyesight}

When creating models and simulations of human perception a significant challenge is figuring out how to test whether the simulation agrees with human judgements. For testing the virtual eyes it would be easy to create a stimulus, and then test how well the virtual eyes perceive the stimulus. Unfortunately, that could easily lead to cases where the eye model and simulation are optimized for properties of the stimulus. An additional concern is it is known that subtle variants in a stimulus can lead to significant differences in human perception. An important criteria for testing digital human models of human perception is: $B e$ fore testing a virtual human with a stimulus, the perceptual properities of the stimulus need to be well understood and established for humans. 


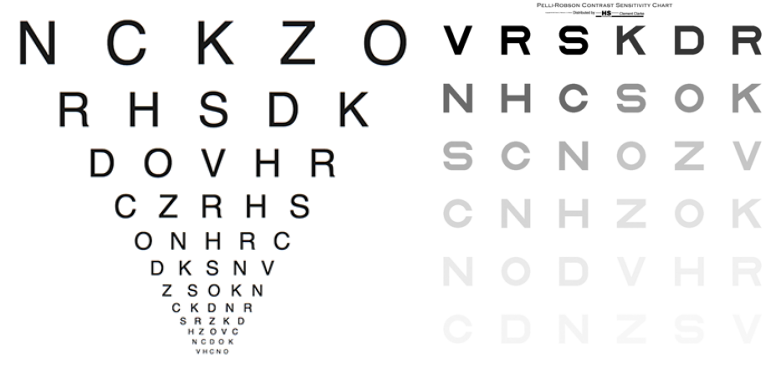

Fig. 5. ETDRS Eye Chart.
Fig. 6. Pelli-Robson Contrast Sensitivity Chart.

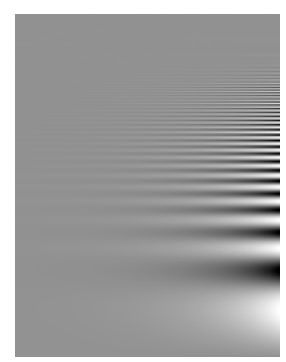

Fig. 7. Campbell-Robson Contrast Sensitivity Chart.

For testing the digital human models of eyesight we use three different eye charts, which test different aspects of human vision. The creation and validation of eye charts is a sub-field in optometry and vision science. Designing eye charts that do not have any subtle perceptual flaws is tricky, as subtle flaws do lead to incorrect evaluations of peoples' eyesight.

We tested the Individual Observer Model of eyesight with the three different eye charts shown in Figure 5, 6 \& 7 . These eye charts test different related facets of human vision that are known to effect peoples' ability to see visual information. Even though the range of visual features on the eye charts is limited (varying letters, size, contrast \& lines), it is well established that human performance on these eye charts is a good predictor of human performance at many visual tasks $[13,8]$.

The chart in Figure 5 is called the ETDRS Chart [5] and it tests how well people can see increasingly smaller letters. People can see the top of the chart easier than the bottom. Shown in Figure 6 is the Pelli-Robson Contrast Sensitivity Chart [15], which shows increasingly harder to see letters, where letter hardness increases from left to right going downwards. The letters become harder to see because of reduced contrast between letter color and background color. Figure 7 shows the Campbell-Robson Contrast Sensitivity Chart [2], which tests the combination of varying size and contrast. When looking at the CampbellRobson Chart observers see the visual detail in the region on the right of the chart. As the observers' visual acuity increases or decreases the size and position of the region they can see detail in either moves up (better vision) or down (worse vision) and gets bigger (better vision) or smaller (worse vision).

\subsection{Results Of Evaluation Of 3600 Virtual Eyes}

Each eye chart is independently used as a stimulus, and each eye chart is divided into a range of equal sized regions. Depending on how each eye chart is used to test human vision, we expect that the predictor $\left(P E R S_{v a}\right)$ correctly identifies what regions are easier to see when compared to other regions within the same eye chart.

For the evaluation 3600 virtual eyes viewed the eye charts and the resulting $P E R S_{v a}$ scores were averaged for all the eyes. If the virtual eyes are effective, then they should agree with human judgements for the eye charts. 


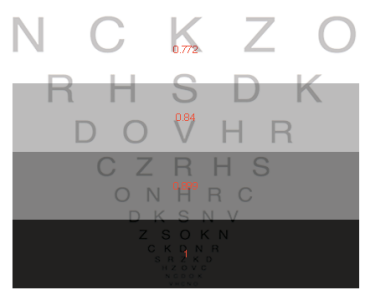

Fig. 8. Heatmap of ETDRS eye chart when divided into 1 by 4 regions.

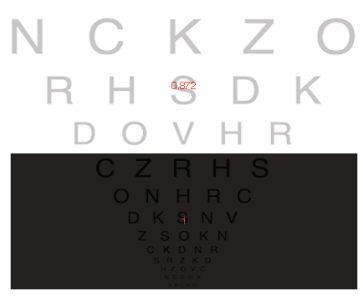

Fig. 11. Heatmap of ETDRS chart divided into 1 by 2 regions.

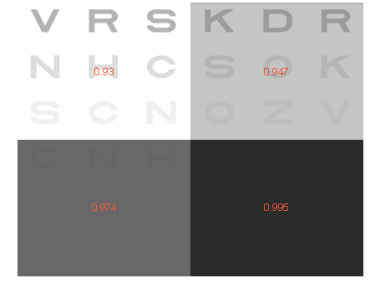

Fig. 9. Heatmap of Pelli- Fig. 10. Heatmap of Robson eye chart when di- Campbell-Robson chart when vided into 2 by 2 regions. divided into 4 by 4 regions.
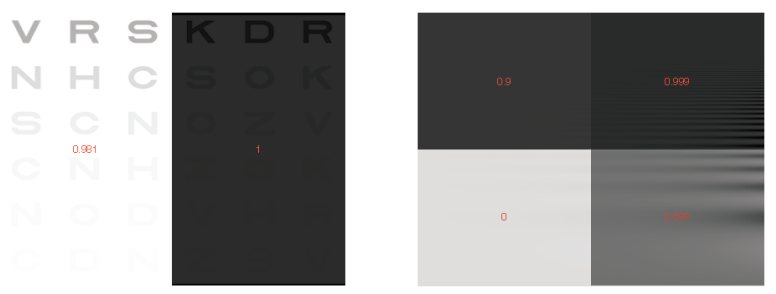

Fig. 12. Heatmap of Fig. 13. Heatmap of Pelli-Robson chart di- Campbell-Robson chart when vided into 2 by 1 regions. divided into 2 by 2 regions.

For the eye charts we find that the predictions of what the virtual eyes see agrees with what humans see. Results are shown in Figure 8 to Figure 13. These figures show how the predictor scores (normalized $P E R S_{v a}$ ) compare between regions. When a region is colored white, it is the easiest to see (lowest normalised $P E R S_{v a}$ score), black indicates the hardest to see (highest $P E R S_{v a}$ score) and grayscale indicates intermediate hardness / PERS $S_{v a}$ score.

ETDRS Chart In Figure 8 we see that the top of the eye chart is white, indicating it is seen easiest, while the bottom of the eye chart is black, indicating it is the hardest part of the eye chart to see. These results are correct.

Are the results due to how the eye chart is divided into 1 by 4 regions? That is addressed by also analysing the eye chart divided into 1 by 2 (Figure 11) and 1 by 3 regions. The results are also in agreement with human vision. That is the virtual eyes find top of the eye chart easier to see, with it becoming increasingly harder to see visual information towards the bottom of the eye chart.

Pelli-Robson Chart We find that the virtual eyes see the Pelli-Robson Chart correctly. Shown in Figure 9 are the results, when the eye chart is divided into 2 by 2 regions. The eye chart gets harder to see from left to right going downwards, where the lower right corner of the chart is the hardest to see. When the eye chart is divided into 2 by 1 (Figure 12) and 2 by 3 regions the results are also in agreement with human vision. 


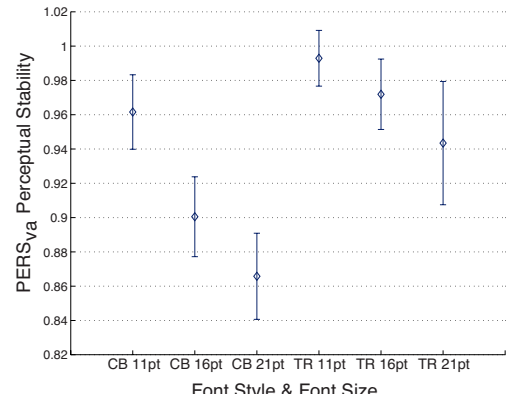

Fig. 14. Virtual eye comparison of font styles \& sizes. Shown is mean \& standard deviation of $P E R S_{v a}$ scores.

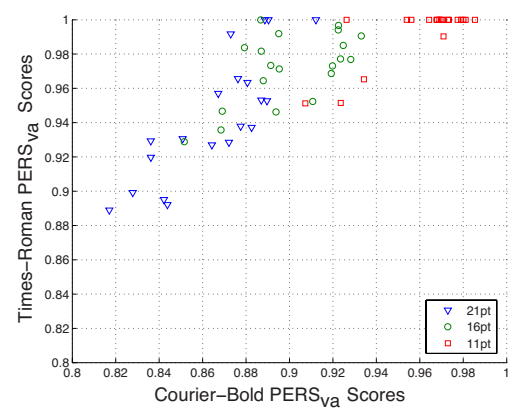

Fig. 15. Scatterplot showing how each virtual eye sees each font \& font size. Each data point is a virtual eye.

Campbell-Robson Chart An especially interesting result is how the virtual eyes see the Campbell-Robson Chart. It is especially interesting because the eye chart does not use letters, rather it uses a more visually complex pattern. Our virtual eye models do see the Campbell-Robson Chart correctly.

When the chart is divided into 4 by 4 regions, results shown in Figure 10, we find the top right of the chart is hardest to see, while the lower right is easiest to see. Nothing is seen on the left side of the chart, as we would expect from human judgements. When the chart is divided into 1 by 2,1 by 3,1 by 4,2 by 2 (Figure 13) and 3 by 3 the virtual eyes are in agreement with how humans see.

\section{Demonstration: Looking At Fonts \& InfoVis Graphs}

Briefly, we describe two examples of the virtual eyes evaluating how easy it is to see different text font styles and different visual styles of graphs. Many fonts styles are often used in visual designs, but it is usually unclear whether one font style is easier to see than another. Especially interesting is whether a particular font style is best for one individual, while a different font style is best for another person. An equivalent challenge for visual analytics and information visualization is correctly designing the visual style line or graph node so that it is easy to see.

Comparing Font Styles Two different font styles are compared, at three different font sizes. Twenty virtual eyes looked at a paragraph of text drawn with the different fonts and at the different sizes. The first font style is CourierBold (CB) font, the second font is Times-Roman (TR), and the 3 font sizes are 11pt, $16 \mathrm{pt}$ and $21 \mathrm{pt}$.

Shown in Figure 14 are the results of the twenty virtual eyes looking at the fonts. Based on the $P E R S_{v a}$ scores, the virtual eyes predict that the CourierBold point size 21 (CB 21pt) is the easiest font to see, while the hardest font to see is Times-Roman 11pt (TR 11pt). In Figure 15 we can check how easily the same eye sees CB versus TR fonts. For example, in Fig 15 the lower left blue triangle indicates a specific virtual eye had a $P E R S_{v a}$ score of approximately 

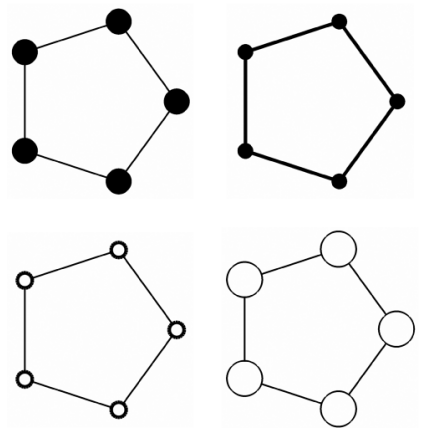

Fig. 16. Four different ways to draw the same simple graph. Nodes and lines vary.

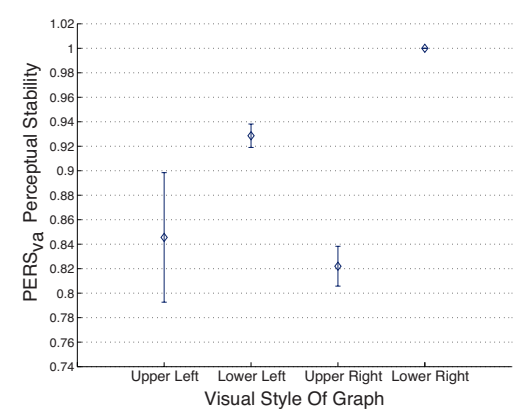

Fig. 17. Comparison of graphs, mean \& std of PERS $S_{v a}$ scores for graphs in Figure 16 .

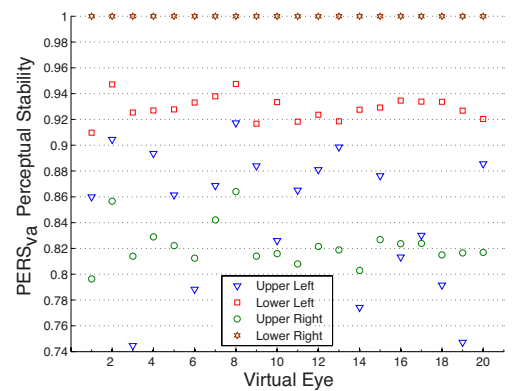

Fig. 18. Distribution of $P E R S_{v a}$ for each virtual eye for each graph style.

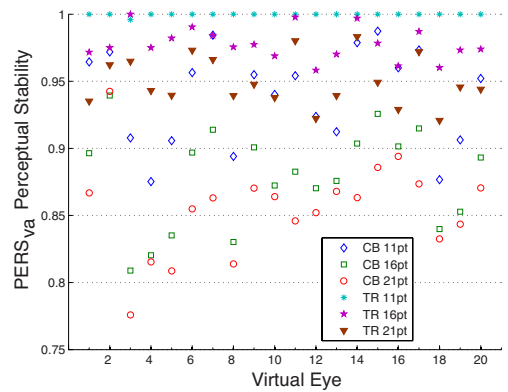

Fig. 19. Distribution of $P E R S_{v a}$ score for each virtual eye for each font style \& size.

0.815 for the CB 21pt font, while the same eye had a $P E R S_{v a}$ score of 0.89 for the TR 21pt font.

Particularly noteworthy is that these results are in agreement with the work of Mansfield et al [11], who evaluated how legible human subjects find the CB versus TR fonts. Figure 19 shows how each individual eyes see each font, these individual differences are discussed in the Discussion section.

Comparing Graph Styles Shown in Figure 16 are the four different simple graphs that twenty virtual eyes looked at. The results shown in Figure 17 indicate that the upper left and upper right graphs are the easiest to see.

Interestingly in Figure 17 the upper left graph has a wider standard deviation, which indicates that due to the differences between the virtual eyes there is more variability between people in how the upper left graph is seen when compared to the upper right graph. Figure 18 shows how each individual eye sees each graph, which is discussed below. 


\section{Discussion \& Conclusions}

In the Introduction we asked Can we model the (users) physiological differences and use the models to adapt and personalize designs, user interfaces and artifacts? In this work we have successfully addressed that question. By showing how to model individual physiological differences in eyesight, then demonstrating using the models (along with $P E R S_{v a}$ ) to evaluate a set of visual designs. After which, the best scoring visual design for a user's eyesight is identified and can be shown to a user, i.e. personalizing the visual design by picking from a set of competing visual designs.

Also posed in the Introduction was the related question How important are these physical differences between people? Or framing it another way, are there benefits by personalizing based on individual differences in users' physiology? The results in Figure 18 \& 19 establishes that the individual differences between people do matter, though the extend to which they matter depends on the visual design. For some visual designs the physical differences between people matter more, for other designs they matter less. For an example of when the physical differences do matter - in Figure 18 most of the virtual eyes find it easiest to see the Upper Right graph (green circle) in Figure 16, while some of the eyes find it easier to see the Upper Left graph (blue triangle). In Figure 19 we find that the physical differences between individual eyes matter less, as all the virtual eyes agree that the Courier-Bold 21pt (red circle) is easiest to see.

An interesting limitation of this work is that our eye model is of low-level early stage vision - predominately concerned with modeling the optics of the human eyeball. There are other differences in human vision which may be worth modeling, e.g. light receptor density and distribution, perception of motion, optical flow, visual crowding [17].

There are also many opportunities for creating Individual Observer Models for various modalities of human experience, e.g. taste, smell, touch. Though creating Individual Observer Models is challenging because they require quantifying the relationship between a sensation and the perception of the sensation. While also requiring the creation of physiologically valid models of human bodies, and requiring that the Individual Observer Models model individual differences in physical function of the human body. Based on previous user modeling research, there are various user models that quantify the perception of designs and artifacts, so there may be opportunities to tie existing models of perception to individual physiological models of users.

Acknowledgements This research was made possible with support from University College Dublin, SICSA - Scottish Informatics and Computer Science Alliance, Stanford Center for Image Systems Engineering, and Microsoft.

\section{References}

1. M. Bennett. Designing For An Individual's Eyes: Human-Computer Interaction, Vision And Individual Differences. PhD thesis, College of Engineering, Mathematical And Physical Sciences, University College Dublin, Ireland, 2009.

2. F. W. Campbell and J. G. Robson. Application of fourier analysis to the visibility of gratings. Journal of Physiology, 197:551-566, 1968. 
3. D. B. Chaffin. Digtal human models for workspace design. Reviews of Human Factors and Ergonomics, 4:44-74, 2008.

4. L. M. Dong, B. S. Hawkins, and M. J. Marsh. Consistency between visual acuity scores obtained at different distances. Archives of Ophthalmology, 120:1523-1533, Nov 2002.

5. F. Ferris, A. Kassoff, G. Bresnick, and I. Bailey. New visual acuity charts for clinical research. American Journal of Ophthalmology, 94:91-96, 1982.

6. J. Freyne and S. Berkovsky. Recommending food: Reasoning on recipes and ingredients. User Modeling, Adaption, and Personalization, 6075:381-386, 2010.

7. H. Fuller, M. Reed, and Y. Liu. Integrating physical and cognitive human models to represent driving behavior. Proceedings of Human Factors and Ergonomics Society 54th Annual Meeting, pages 1042-1046, 2010.

8. A. Ginsburg, D. Evans, R. Sekuler, and S. Harp. Contrast sensitivity predicts pilots' performance in aircraft simulators. American Journal of Optometry and Physiological Optics, 59:105-108, 1982.

9. R. R. Krueger, R. A. Applegate, and S. M. MacRae. Wavefront Customized Visual Correction: The Quest for Super Vision II. 2004.

10. Y. Liu, R. Feyen, and O. Tsimhoni. Queueing network-model human processor (qnmhp): A computational architecture for multitask performance in human-machine systems. ACM Transactions on Computer-Human Interaction, 13(1):37-70, 2006.

11. J. Mansfield, G. Legge, and M. Bane. Psychophysics of reading: Xv. font effects in normal and low vision. Journal of Investigative Ophthalmology and Visual Science, 37(8):1492-1501, 1996.

12. S. Marsella. Modeling emotion and its expression in virtual humans. User Modeling, Adaption, and Personalization, 2010.

13. T. T. Norton, D. A. Corliss, and J. E. Bailey. The Psychophysical Measurement of Visual Function. 2002.

14. S. E. Palmer. Vision Science: Photons to Phenomenlogy. 1999.

15. D. G. Pelli, J. G. Robson, and A. J. Wilkins. The design of a new letter chart for measuring contrast sensitivity. Clinical Vision Sciences, 2(3):187-199, 1988.

16. M. Reed, J. Faraway, D. B. Chaffin, and B. J. Martin. The humosim ergonomics framework: A new approach to digital human simulation for ergonomic analysis. Digital Human Modeling for Design and Engineering Conference, SAE Technical Papers Series, 2006.

17. R. Rosenholtz, Y. Li, J. Mansfield, and Z. Jin. Feature congestion: A measure of display clutter. Proceedings of SIGCHI, pages 761-770, 2005.

18. T. O. Salmon and C. van de Pol. Normal-eye zernike coefficients and root-meansquare wavefront errors. Journal of Cataract Refractive Surgery, 32:2064-2074, Dec 2006.

19. L. N. Thibos, R. A. Applegate, J. T. Schwiegerling, R. Webb, and V. S. T. Members. Standards for reporting the optical aberrations of eyes. Vision Science and its Applications, pages 232-244, Feb 2000.

20. L. N. Thibos, A. Bradley, and X. Hong. A statistical model of the aberration structure of normal, well-corrected eyes. Journal of Ophthalmic and Physiological Optics, 22:427-433, 2002.

21. S. K. West, B. Muñoz, G. S. Rubin, O. D. Schein, K. Bandeen-Roche, S. Zeger, P. S. German, and L. P. Fried. Function and visual impairment in a population based study of older adults. Journal of Investigative Ophthalmology and Visual Science, 38(1):72-82, 1997. 